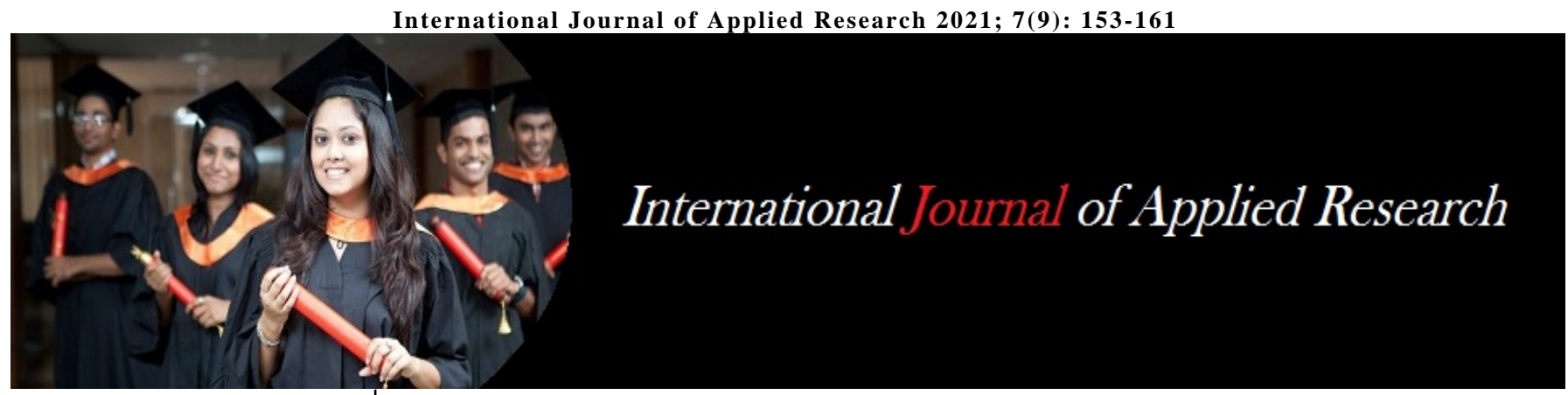

ISSN Print: 2394-7500 ISSN Online: 2394-5869 Impact Factor: 8.4 IJAR 2021; 7(9): 153-161 www.allresearchjournal.com

Received: 10-07-2021 Accepted: 12-08-2021

Alisha Khokhawala Modern College of Physiotherapy, Shivaji Nagar, Pune, Maharashtra, India

Dr. Gauri Mayank Afle Professor, Department of Cardiovascular and Respiratory Physiotherapy, Modern College of Physiotherapy, Pune, Maharashtra, India

Corresponding Author: Alisha Khokhawala Modern College of Physiotherapy, Shivaji Nagar, Pune, Maharashtra, India

\section{Effect of ELTGOL on pulmonary function tests in patients with COPD: A quasi experimental study}

\author{
Alisha Khokhawala and Dr. Gauri Mayank Afle
}

DOI: https://doi.org/10.22271/allresearch.2021.v7.i9c.8966

\section{Abstract}

Background: COPD is defined as a disease state characterized by progressive development of chronic airflow limitation that is not fully reversible and includes chronic bronchitis, emphysema and smaller airway disease. Pulmonary function testing (PFT) reveals airflow obstruction on spirometry that manifests as a reduced forced expiratory flow in one second (FEV1)/forced vital capacity (FVC) ratio (FEV1/FVC). Airway mucus hypersecretion is one of the most important features of COPD which is manifested as cough with expectoration. Chronic cough and sputum production are a few key features of chronic obstructive pulmonary disorder. These occur due to mucus hypersecretion and ciliary dysfunction. Airway clearance techniques (ACTs) involve the application of forces to enhance sputum removal from the airways. ELTGOL is known to be effective in mucus clearance in patients with COPD, chronic bronchitis, bronchiectasis etc. The goal of ELTGOL is to control the expiratory flow to prevent airway closure and facilitate mucus clearance to avoid airway compression. There are various studies that show the positive effects of airway clearance techniques on pulmonary functions. The benefits of ELTGOL on mucus clearance have also been proved. But the effects of ELTGOL on the pulmonary functions have not yet been proven to the best of our knowledge. Therefore, this study will check the effects of ELTGOL on pulmonary functions in patients with COPD. 33 Subjects were selected and explained about the intervention. Pre intervention assessment using PFT was done and intervention was given for 2 weeks once a day for 30 minutes. Post intervention PFT was done and the data was then analysed.

Result: On comparison of pre and post data, change in FVC score was from $2.4 \pm 0.37$ to $3.04 \pm 0.39$, change in FEV1 score was from $1.43 \pm 0.27$ to $2.36 \pm 0.29$, change in PEFR score was from $3.38 \pm 0.66$ to $4.35 \pm 0.7$ and change in FEV1/FVC was from $0.58 \pm 0.04$ to $0.76 \pm 0.05$. This shows a statistically significant difference in the pre and post PFT scores.

Conclusion: On the basis of the result, we can conclude that ELTGOL is effective in improving pulmonary functions in patients with moderate to severe COPD.

Keywords: chronic obstructive pulmonary disease, airway clearance techniques, pulmonary function tests, ELTGOL

\section{Introduction}

According to GOLD Initiative for Chronic Obstructive Pulmonary Disorders, COPD is defined as a disease state characterized by progressive development of chronic airflow limitation that is not fully reversible and includes chronic bronchitis, emphysema and smaller airway disease ${ }^{[1]}$. It is estimated by WHO that over 200million people worldwide suffer from COPD and it is the leading cause of death in the world ${ }^{[2,3]}$. Overall, the prevalence of COPD is between $3-10 \%$ but this varies widely depending on the diagnostic criteria used ${ }^{[4]}$. According to the US National Institute of Health (NIH) in the year 2000 an estimated 10 million Americans had self-reported doctor diagnosed COPD. They also noted that National Health and Nutrition Examination Survey III estimated that 24 million American adults have evidence of airflow limitation ${ }^{[4]}$.

India has $18 \%$ of the global population and an increasing burden of chronic respiratory disease ${ }^{[5]}$. Prof. Lalit Dandona published an article which stated that the number of COPD cases in Indian increased from 28.1 million in 1990 to 55.3 million in 2016, an increase in prevalence from $3.3 \%$ to $4.2 \%{ }^{[5]}$.

Studies have shown that COPD may be underdiagnosed and undertreated in older patients ${ }^{[4]}$. 
COPD has been considered a diagnosis that includes either chronic bronchitis or emphysema or an overlap of both ${ }^{[4]}$. Recent guidelines have attempted to standardize the definition of COPD using findings of incompletely reversible airflow obstruction on spirometry ${ }^{[4]}$. Smoking is one of the greatest risk factors for the development of COPD. Other factors such as environmental and inhalational exposures to gases, dust and pollution also contribute to development of COPD ${ }^{[4]}$.

Radiologically lungs demonstrate hyperinflation and also show emphysematous changes such as bullae and blebs ${ }^{[4]}$. Pulmonary function testing reveals airflow obstruction on spirometry that manifests as a reduced forced expiratory flow in one second (FEV1)/forced vital capacity (FVC) ratio (FEV1/FVC). The severity of COPD is based on the accelerated decline in FEV1 with age ${ }^{[4,3]}$.

Airway mucus hypersecretion is one of the most important features of COPD which is manifested as cough with expectoration [2]. COPD exacerbations increase airway inflammation and this plays a part in the progressive decline in FEV1 ${ }^{[3]}$.

A 12year study conducted by Sheeman showed that there was a relation between chronic mucus production and FEV1 decline after adjustment for age and smoking ${ }^{[2,6]}$. Vestbo et al. did a 5 year follow up study on 5345 women and 4081 men proving that chronic airway mucus hypersecretion was significantly associated with FEV1 decline in COPD patients ${ }^{[2,7]}$.

Other than declining FEV1 and FEV1/FVC ratio, a descending FVC is also a common lung function manifestation on COPD. A drop in FVC results in increased residual volume which also leads to lung hyperinflation ${ }^{[9]}$.

Chronic cough and sputum production are a few key features of chronic obstructive pulmonary disorder. These generally occur due to mucus hypersecretion and ciliary dysfunction. Airway clearance techniques (ACTs) involve the application of forces to enhance sputum removal from the airways ${ }^{[10]}$. There's evidence that suggests the role of airway clearance techniques in preserving pulmonary functions ${ }^{[10]}$. There's evidence that suggests the role of airway clearance techniques in preserving pulmonary functions ${ }^{[10]}$. A recent consensus statement describes ACTs as a cornerstone of management for adults with cystic fibrosis and bronchiectasis ${ }^{[10]}$. A Cochrane review that included 7 trials of 'bronchopulmonary hygiene physical therapy' in COPD and bronchiectasis found evidence to support increased sputum production and clearance from the lungs ${ }^{[10]}$.

There is lack of evidence on the superiority of any particular ACT or device in patients with chronic bronchitis, COPD and bronchiectasis ${ }^{[11]}$. ACTs consist of various approaches such as forced exhalation, manual compression, vibrations, positive expiratory pressure devices, huffing and shaking [11, 2]. A study by Elisabeth Westerdhal in 2019 showed that physical therapists generally prescribe PEP based ACTs for patients having an acute exacerbation of COPD. This study was done in Sweden ${ }^{[12]}$. The main factors influencing the choice of ACTs were the degree of dyspnea, work of breathing and access to equipment ${ }^{[12]}$ There are studies that present a positive effect of Flutter when compared with a sham group ${ }^{[13]}$. Airway clearance techniques improve the quality of life and reduce morbidity and mortality of patients suffering from chronic bronchitis or bronchiectasis ${ }^{[14]}$.

One such airway clearance technique known as ELTGOL is known to be effective in mucus clearance in patients with COPD, chronic bronchitis, bronchiectasis etc. ${ }^{[15}{ }^{16]}$. In slow expiration with glottis open in lateral posture the patient lies in lateral decubitus position and breathes out slowly through an open glottis from the Functional Residual Capacity to Residual Volume ${ }^{[16]}$. The goal of this technique is to control the expiratory flow to prevent airway closure and facilitate mucus clearance to avoid airway compression ${ }^{[16,17]}$. Martins et al. reported an increase in mucus clearance in the peripheral area in subjects with COPD after ELTGOL by radio aerosol inhalation [17, 18]. A study done by Gerard Munoz et al. concluded that application of ELTGOL twice daily over 1 year facilitated secretion removal, improved quality of life and was associated with fewer exacerbations in patients suffering from bronchiectasis ${ }^{[19]}$. Herrero Cortina et al. compared three ACTs ie. autogenic drainage, ELTGOL and temporary positive expiratory pressure breathing in sputum expectoration in bronchiectasis patients. They concluded that slow expiratory ACTs enhance mucus clearance during treatment sessions and reduce expectorations for the rest of the day in patients with bronchiectasis ${ }^{[20]}$.

There are various studies and evidence that shows the positive effects of airway clearance techniques on pulmonary functions. The benefits of ELTGOL on mucus clearance has also been proved. But the effects of ELTGOL on the pulmonary functions have not yet been proven to the best of our knowledge.

Therefore, this study will check the effects of ELTGOL on pulmonary functions in patients with COPD.

\section{Materials and Methods}

The type of the study design was a Quasi Experimental Study. The subjects were selected from various hospitals in and around the city after a thorough consultation with the physicians based on the inclusion and exclusion criteria. Both males and females between the age group of 45-75 years were included in the study. Patients with FEV1 less than $70 \%$ as predicted from age and height, patients with a ratio of FEV1/FVC less than 0.7 and patients with moderate to severe COPD according to GOLD criteria were included int the study. Any patient who had an exacerbation during the study, who's saturation dropped below 85\%, patients having cardiovascular, neuromuscular or musculoskeletal involvements or chest wall deformities and patients who had undergone any cardiothoracic surgery were excluded from the study. Convenient sampling method was used to collect a sample size of 33 patients (19 males and 14 females). The duration of the study was one year. Informed consent was taken from the patients. The demographic data and the basic personal information, vital parameters, level of dyspnoea, co-morbidities and information regarding current medication of each patient was taken. Pre intervention assessment using pulmonary function tests ie. Forced Vital Capacity (FVC), Forced expiratory Volume in one second (FEV1), the ratio of Forced Vital Capacity and forced Expiratory Volume (FEV1/FVC) and Peak Expiratory Flow Rate (PEFR) were done and obtained. Intervention was given for 2 weeks once a day for 30 minutes. Post intervention pulmonary function tests were done and obtained. Data was analysed using Paired T-test (within sample) for the pre and post score recorded and conclusion was drawn.

The materials used during the study were a demographic 
data sheet, a consent form, a foam mat, a stethoscope and pulmonary function tests (FVC, FEV1, FEV1/FVC ratio and PEFR).

\section{Results and Discussion}

Result: A total of 33 patients ie. 19 males and 14 females, suffering from moderate to severe COPD in the age group of 50-60 years volunteered to participate in the study and completed 2 weeks of the intervention period.

The mean age of the experimental group was $58.1 \pm 4.8$.

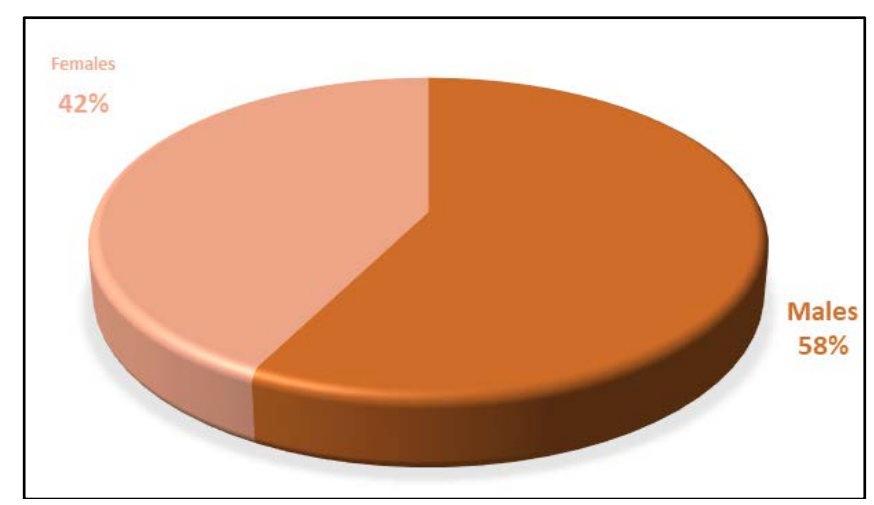

Graph 1: Pie chart showing gender wise distribution for participants in the study

\section{Comparison of FVC Score}

The Forced Vital Capacity is a measure of air the lungs can hold and is highly dependent on the amount of force used by the patient in early expiration. It is generally reduced in both obstructive and restrictive conditions.
The pre and post intervention data was analysed using Paired $\mathrm{T}$ test and there was a significant difference in pre $(2.4 \pm 0.37)$ and post $(3.04 \pm 0.39)$ scores of Forced Vital Capacity after 2 weeks of intervention ie. ELTGOL.

Table 1: Comparison of pre and post score for FVC after 2 weeks of Eltgol

\begin{tabular}{|c|c|c|c|c|c|c|c|}
\hline Parameter & \multicolumn{2}{|c|}{ Pre } & \multicolumn{2}{c|}{ Post } & T Value & p Value & Result \\
\hline \multirow{2}{*}{ FVC } & Mean & SD & Mean & SD & & & \\
\cline { 2 - 5 } & 2.4 & 0.37 & 3.04 & 0.39 & -6.85 & $0.000^{*}$ & Significant \\
\hline
\end{tabular}

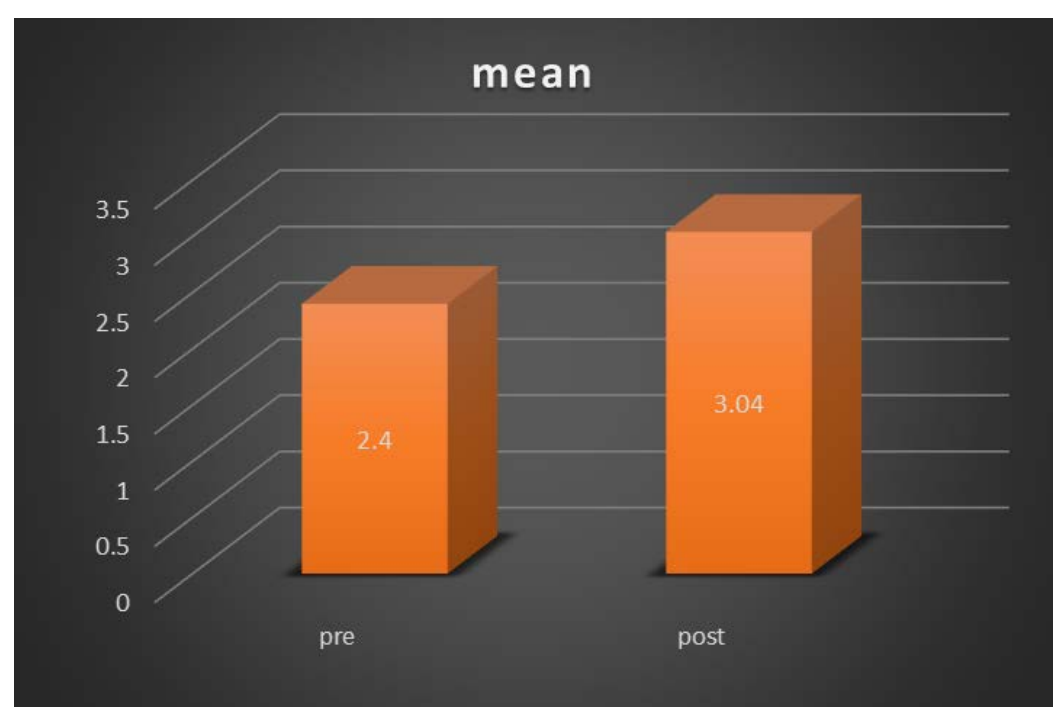

Graph 2: Bar diagram showing comparison of pre and post FVC scores

\section{Comparison of FEV1 Score}

The Forced Expiratory Volume in one second is a measure of how easily air flows through the lungs and reflects airflow in the larger airways. COPD patients often have narrowing and inflammation of the airways and this hinders how fast the air can leave the lungs. This in turn leads to a fall in FEV1.
The pre and post intervention data was analysed using Paired $\mathrm{T}$ test and there was a significant difference in pre $(1.43 \pm 0.27)$ and post $(2.36 \pm 0.29)$ scores of Forced Expiratory Volume in one second after two weeks of intervention ie. ELTGOL.

Table 2: Comparison of pre and post score for FEV1 after 2 weeks of Eltgol

\begin{tabular}{|c|c|c|c|c|c|c|c|}
\hline Parameter & \multicolumn{2}{|c|}{ PRE } & \multicolumn{2}{c|}{ POST } & T Value & p Value & RESULT \\
\hline \multirow{2}{*}{ Fev1 } & MEAN & SD & MEAN & SD & & & \\
\cline { 2 - 5 } & 1.43 & 0.27 & 2.36 & 0.29 & -17.57 & $0.001^{*}$ & SIGNIFICANT \\
\hline
\end{tabular}




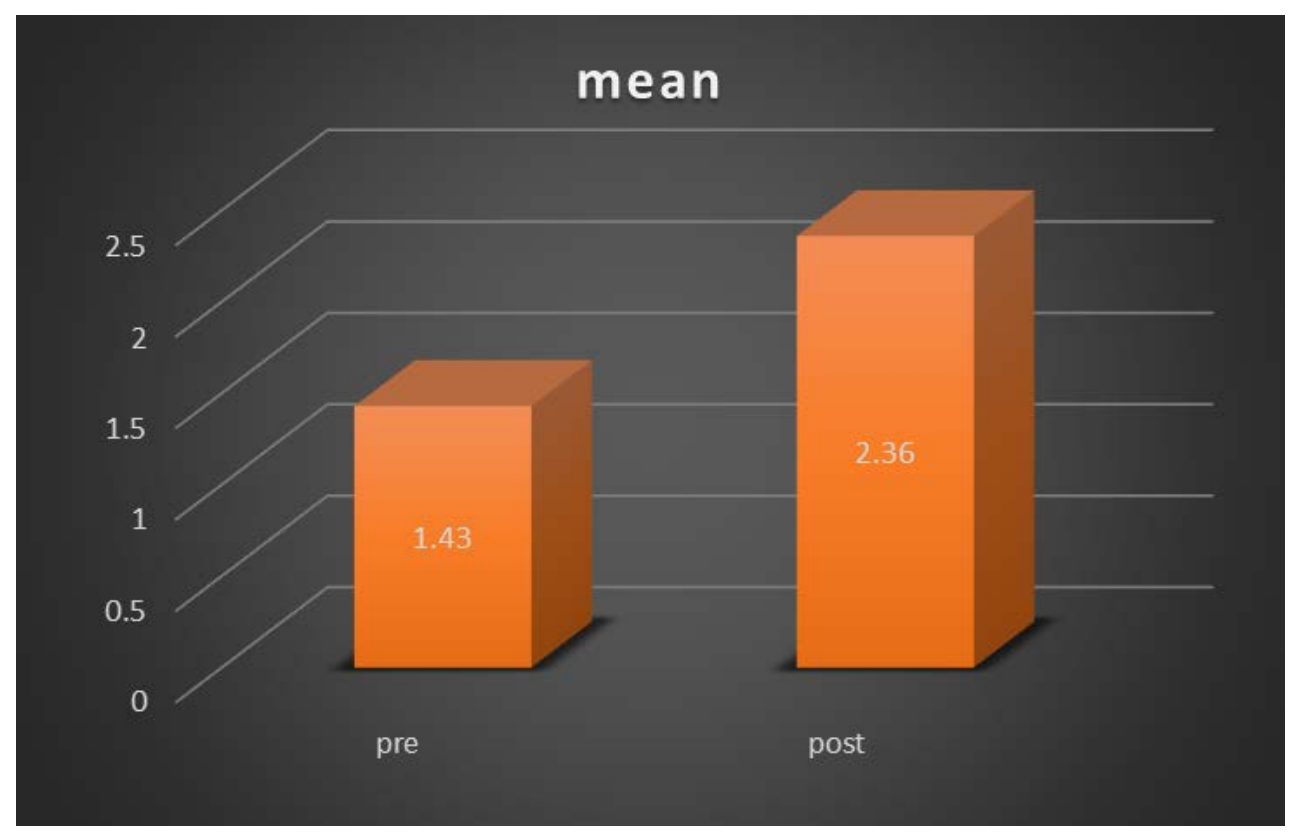

Graph 3: Bar diagram showing comparison of pre and post FEV1 scores

\section{Comparison of PEFR Score}

Peak Expiratory Flow Rate is the maximum flow that occurs at any given point in time during the FVC.
The pre and post intervention data was analysed using Paired $\mathrm{T}$ test and there was a significant difference in pre (3.38 \pm 0.66$)$ and post $(4.35 \pm 0.7)$ scores of Peak Expiratory Flow Rate after two weeks of intervention ie. ELTGOL.

Table 3: Comparison of pre and post score for PEFR after 2 weeks of Eltgol

\begin{tabular}{|c|c|c|c|c|c|c|c|}
\hline Parameter & \multicolumn{2}{|c|}{ PRE } & \multicolumn{2}{c|}{ POST } & T Value & p Value & Result \\
\hline \multirow{2}{*}{ PEFR } & Mean & SD & MEAN & SD & \multirow{2}{*}{-16.58} & \multirow{2}{*}{$0.000 *$} & \multirow{2}{*}{ Significant } \\
\cline { 2 - 5 } & 3.38 & 0.66 & 4.35 & 0.70 & & & \\
\hline
\end{tabular}

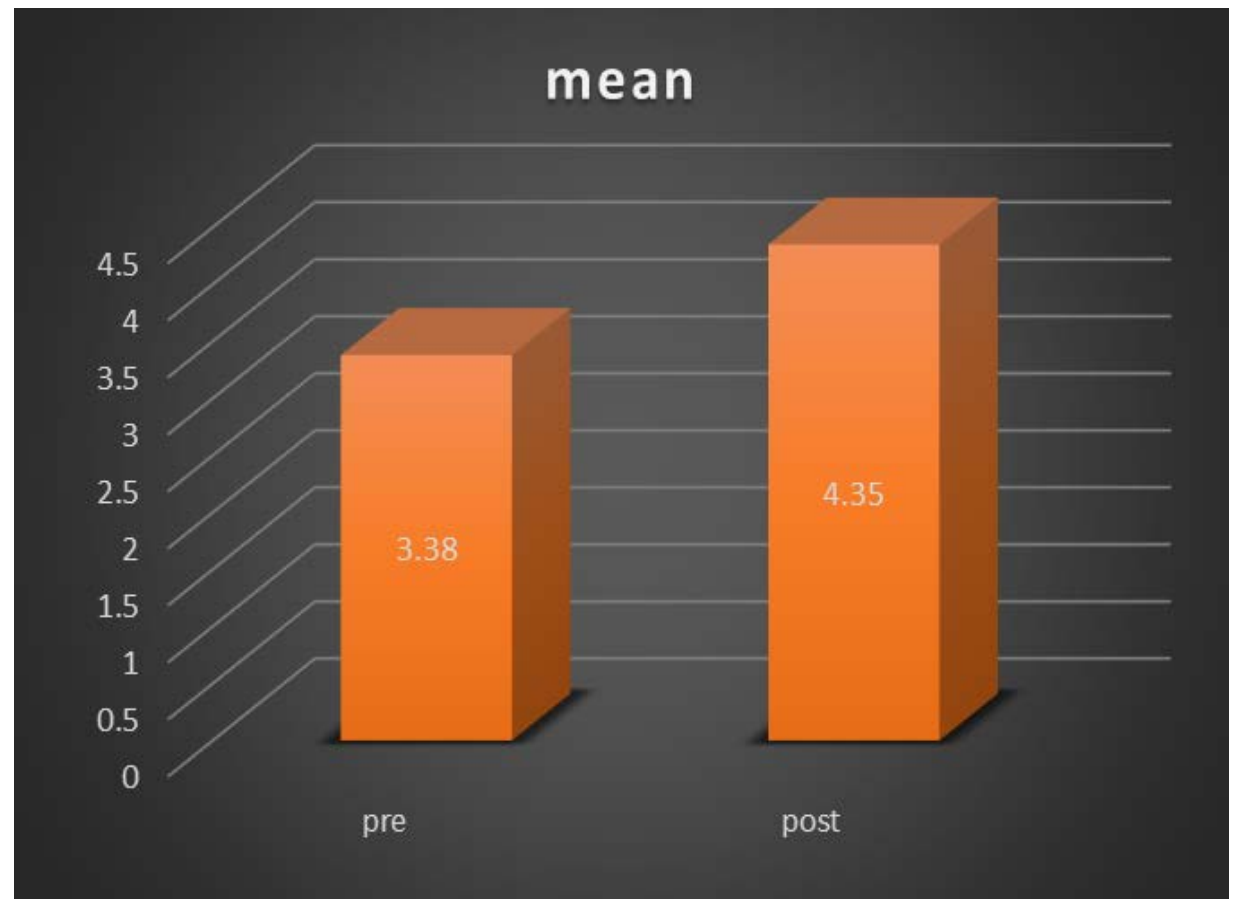

Graph 4: Bar diagram showing comparison of pre and post PEFR scores

\section{Comparison of FEV1/FVC}

If FEV1 is decreased disproportionately to the FVC a diagnosis of COPD is made. To check if the decrease is disproportionate the FEV1/FVC ratio is calculated and a ratio of $<0.7$ after bronchodilator is considered a diagnosis of COPD.
The pre and post intervention data was analysed using Paired $\mathrm{T}$ test and there was a significant difference in pre $(0.58 \pm 0.04)$ and post $(0.76 \pm 0.05)$ scores of the ratio of forced expiratory volume in one second and forced vital capacity after two weeks of intervention ie. ELTGOL. 
Table 4: Comparison of pre and post score for the ratio of FEV1/FVC after 2 weeks of ELTGOL

\begin{tabular}{|c|c|c|c|c|c|c|c|}
\hline Parameter & \multicolumn{2}{|c|}{ PRE } & \multicolumn{2}{c|}{ POST } & T Value & p Value & Result \\
\hline \multirow{2}{*}{ FEV1/FVC } & MEAN & SD & MEAN & SD & \multirow{2}{*}{-22.81} & \multirow{2}{*}{$0.001^{*}$} & \multirow{2}{*}{ Significant } \\
\cline { 2 - 5 } & 0.58 & 0.04 & 0.76 & 0.05 & & \\
\hline
\end{tabular}

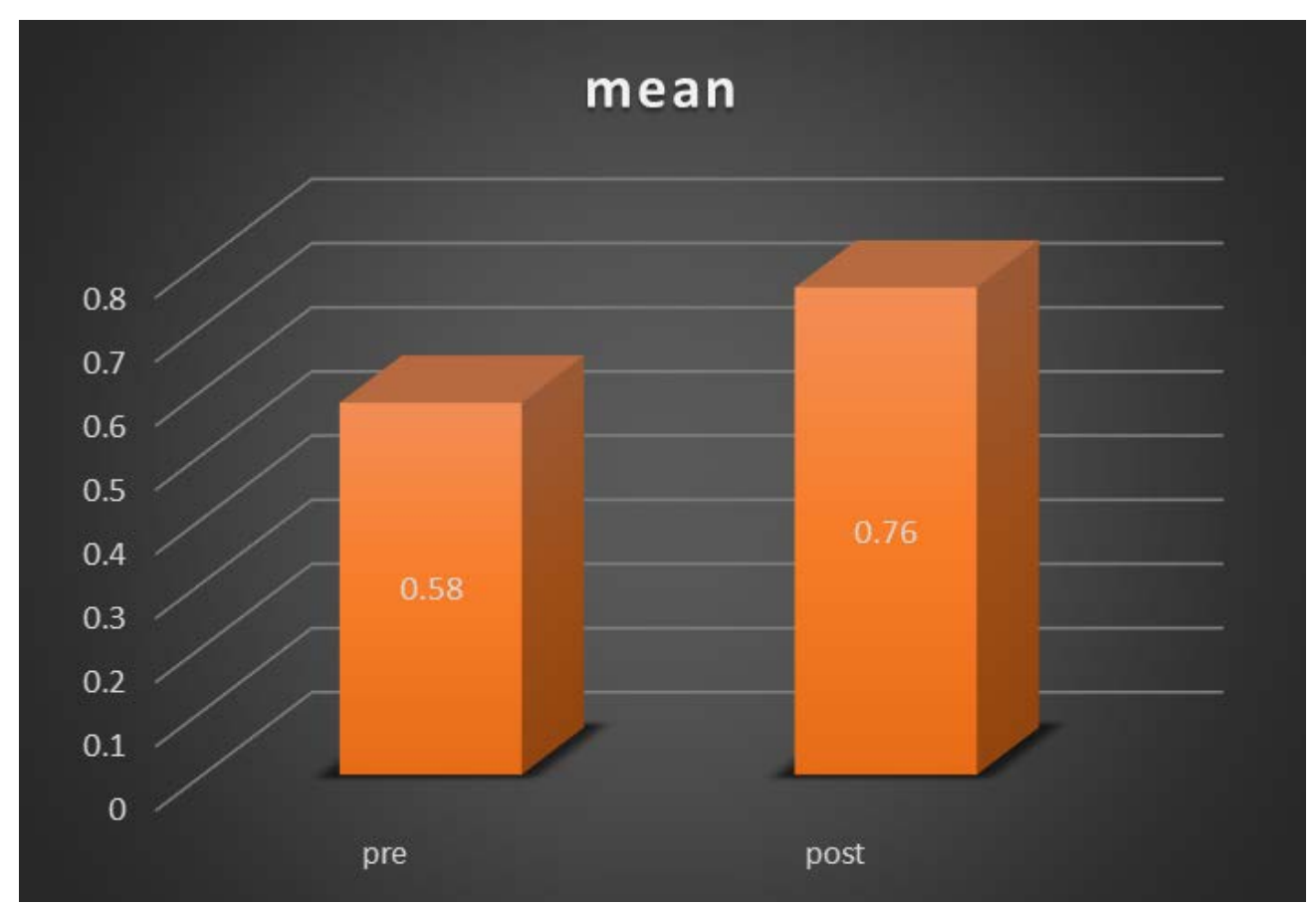

Graph 5: Bar diagram showing comparison of pre and post scores for ratio of FEV1/FVC

The pre and post intervention data was analysed using Paired $\mathrm{T}$ test and there was a significant difference ie. $\mathrm{p}=0.000^{*}$, in pre and post scores of the pulmonary function tests after 2 weeks of intervention ie. ELTGOL.

Table 5: Combined comparison of pre and post score for the Pulmonary Function Tests done after 2 weeks of Eltgol

\begin{tabular}{|c|c|c|c|c|c|}
\hline Parameters & PRE score & POST score & T Value & p Value & Result \\
\hline FVC & 2.4 & 3.04 & -6.85 & $0.000^{*}$ & Significant \\
\hline FEV1 & 1.43 & 2.36 & -17.57 & $0.001^{*}$ & Significant \\
\hline Pefr & 3.38 & 4.35 & -16.58 & $0.000^{*}$ & Significant \\
\hline FEV1/FVC & 0.58 & 0.76 & -22.81 & $0.001^{*}$ & Significant \\
\hline
\end{tabular}

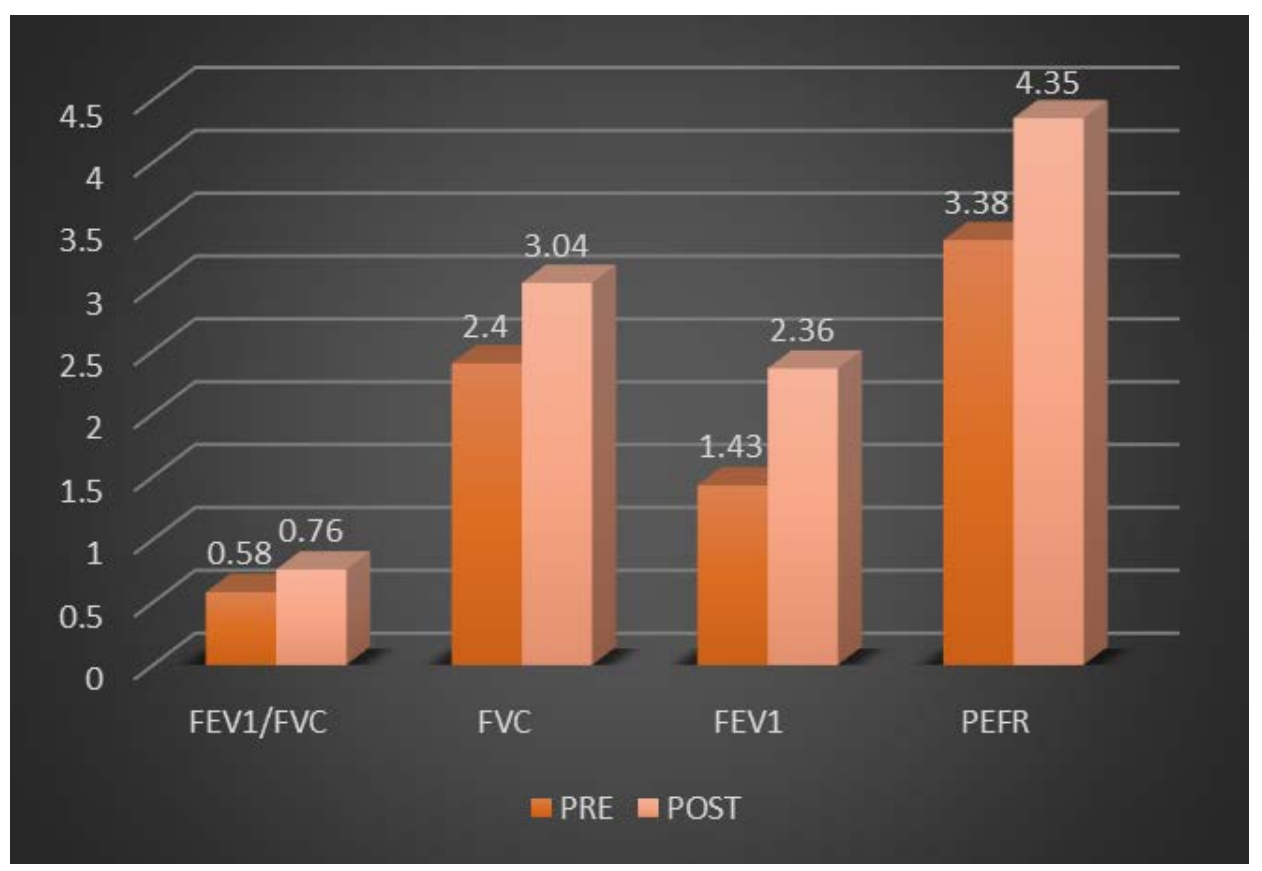

Graph 6: Combined bar diagram showing comparison of pre and post scores for the Pulmonary Function Tests 
This shows that ELTGOL is effective in clearing mucus and improving the pulmonary functions of patients with moderate to severe COPD after 2 weeks of intervention.

\section{Discussion}

The primary aim of this study was to check the effect of ELTGOL on pulmonary function tests in patients with moderate to severe chronic obstructive pulmonary disease. A total of 33 patients both males and females were included in the study based on the inclusion criteria.

In this study we found that ELTGOL is effective in improving the pulmonary functions of patients with COPD.

The results of the present study are in agreement with the findings of Martins et al. who conducted a study in 2012 to check the effect of ELTGOL on mucus clearance in stable patients with chronic bronchitis. They concluded that ELTGOL was efficient in increasing peripheral airway clearance in the dependent lung in patients with chronic bronchitis in mild to moderate COPD ${ }^{[18]}$. They conducted a study in 17 patients with chronic bronchitis. Their main finding was that ELTGOL significantly increased mucus clearance in the peripheral area of the dependent lung, demonstrating the selective aspect of this technique.

Our results were also in accordance with a study conducted by Bellone et al. who compared the efficacy of 3 techniques of chest physiotherapy including ELTGOL. They did the study on 10 patients who had exacerbations of chronic bronchitis. They evaluated the weight of the secretions that were eliminated after performing the 3 chest physiotherapy techniques. They found that during the intervention all 3 techniques were safe and effective in removing the secretions and ELTGOL and Flutter could remove significantly greater quantities of sputum as compared to postural drainage ${ }^{[22,18]}$.

\section{ELTGOL: An Airway Clearance Technique}

ELTGOL stands for Slow Expiration with Glottis open in Lateral Decubitus position. According to the technique ELTGOL promotes a contra gravitational mobilization of the mucus in the peripheral airways ${ }^{[18]}$. When the patient lies in a lateral decubitus position there are 3 forces that act: gravitational force, mediastinal weight and pressure from the abdominal viscera on the infralateral lung ${ }^{[18]}$. A higher deflation of the infralateral lung is achieved through the lateral posture associated with slow expiratory flow, obtained in low lung volume ie. functional residual capacity (FRC) which thus improves the mucus clearance ${ }^{[10,18]}$. The expiratory flow can be optimized in different bronchial generations and low speed flow will provide a more distal action which is influenced by the gas liquid interaction ${ }^{[23,}$ ${ }^{18}$. Thus this slow flow would mobilize mucus more in the distal airways ${ }^{[24,18]}$.

The ELTGOL technique was developed to increase mucus clearance and improve expiratory volume in subjects with chronic lung disease ${ }^{[28,17]}$. This technique is based on the expiratory phase which has to be prolonged and slow ${ }^{[17]}$. Subjects with chronic lung disease usually have dynamic airway collapse ${ }^{\text {[17] }}$. The slow expiration with glottis open performed in ELTGOL increases expiratory time thus allowing more amount of volume of air/secretions to be exhaled [17]. A finding by Fernanda et al. shows that ELTGOL mobilized more than $80 \%$ of expiratory reserve volume in subjects with moderate airway obstruction.
Agnew et al. proposed a model which stated that mucus follows a route from the peripheral area to the central area of the lungs. Therefore, the mucus cleared from the peripheral area is moved to the intermediate area to the central area by a cough ${ }^{[25,24,18]}$. Thus the Agnew model reinforces that ELTGOL was responsible for the increased clearance of mucus from the peripheral area ${ }^{[25,18]}$.

ELTGOL has also been effective in clearing secretions from patients suffering from bronchiectasis and cystic fibrosis. A study conducted by Herrero-Cortina et al. showed the shortterm effects of 3 airway clearance techniques in bronchiectasis and found that autogenic drainage and ELTGOL induced a higher sputum expectoration than temporary positive pressure ${ }^{[20]}$. ELTGOL in combination with an oscillating positive expiratory pressure has shown greater effectiveness in patients with bronchiectasis by Guimaraes et al. ${ }^{[26,20]}$.

A study by Gerard Munoz et al. in 2018 on bronchiectasis patients showed that twice daily ELTGOL technique facilitated secretion removal and was associated with fewer exacerbation improvement in quality of life and reduced cough impact ${ }^{[19]}$.

Guimaraes at al also carried out a study in 2014 on the effect of ELTGOL in adult cystic fibrosis and concluded that ELTGOL promoted higher secretion removal and improvement in airway resistance and conductance than the Flutter valve. These techniques were equivalent in reducing the pulmonary hyperinflation and air trapping in cystic fibrosis ${ }^{[27]}$.

The underlying mechanism of ELTGOL uses airways compression to improve the air-liquid interaction in the peripheral airways and in turn promoting secretion displacement ${ }^{[28]}$. The goal of ELTGOL is to control expiratory flow to prevent airway closure and facilitate mucus clearance in contrast to conventional postural drainage in which mucus clearance is only increased in the dependent inferiorly positioned lung ${ }^{[16]}$.

\section{Mechanism of ELTGOL}

Mucus is cleared from the respiratory tract by two main mechanisms: ciliary transport and expiratory airflow. ELTGOL is based on increasing airflow clearance by a mechanism known as two phase gas liquid flow ${ }^{[16,29]}$. In this model when air flows over mucus it creates a shear force that can overcome the resistive forces in the mucus layer. The higher the velocity of the airflow the greater the shear force ${ }^{[16]}$.

In ELTGOL the volume of the dependent lung is reduced by placing the patient in the lateral decubitus position and by limiting breathing to expiratory reserve volume ${ }^{[16,17]}$. This reduces the total cross-sectional area of the peripheral airways where mucus is primarily produced. Since maximum airflow velocity is inversely proportional to airway diameter, the velocity of the airflow in the peripheral airway is increased ${ }^{[16,30]}$. Airway patency is maintained by increasing intraluminal pressure via slow expiration through an open glottis ${ }^{[16,31]}$.

An investigation done by Rodney G. Benjamin et al. focused on the fact that open airways thickly lined with mucus remained calm in the presence of low airflows. With increased airflow the layer of mucus became unstable such that ripples and waves formed on it. Further increase in airflow caused wave motion of the mucus in the direction of the air flowing over the mucus layer ${ }^{[32]}$. 
The factors affecting transport of mucus by two phase gas liquid flow include the velocity of air passing over the liquid, the thickness of the mucus layer, the viscosity and elasticity of mucus, the ratio of mucus thickness to tube diameter and whether the air flowing over the mucus is unidirectional or cyclical in a bidirectional manner ${ }^{\text {[32] }}$. They also stated that two phase gas liquid flow is of no importance in normal airways which are generally lined with a 5 to 10 micron layer of mucus. In normal airway the mucociliary transport system forms the primary basis for mucus clearance ${ }^{[32]}$.

For the two phase gas liquid flow mechanism to be effective the peak expiratory flow rate (PEFR) must be greater than the peak inspiratory flow rate (PIFR) by more than $10 \%$ so that mucus moves proximally ${ }^{[33]}$. The PEFR must also exceed 30 to $60 \mathrm{~L} / \mathrm{min}$ to overcome the adhesive strength by which the mucus is attached to the interface. During normal tidal breathing at rest the PEFR is not more than 30L/min and PIFR is greater than PEFR; this results in secretions not being mobilized ${ }^{[33]}$.

In ELTGOL the expiratory phase is prolonged as the patient is instructed to slowly breathe out with the glottis open. This increases the peak expiratory flow rate thus enhancing mucus clearance. Overall this results in greater clearance of mucus from the peripheral airways ${ }^{[16,18]}$.

\section{Pulmonary Functions and ELTGOL}

Pulmonary functions are generally affected in patients with COPD. COPD is a disease with pulmonary as well as extra pulmonary effects. The pulmonary component is characterized by airflow limitation that is not fully reversible ${ }^{[32]}$.

Pulmonary function testing has three components: spirometry, lung volumes and diffusing capacity of carbon monoxide. Each of these components are affected in COPD but only spirometry is necessary to make the diagnosis ${ }^{[18]}$. Spirometry consists of forced vital capacity (FVC), forced expiratory volume in one second (FEV1) and the ratio of forced expiratory volume in one second to forced vital capacity (FEV1/FVC) ${ }^{[8]}$. To perform spirometry the patient performs a deep inhalation and then breathes out as fast as they can. This is known as forced exhalation. During this the total lung volume of air the patient can exhale in one breath is called forced vital capacity and the amount of air the person can exhale in one second is called forced expiratory volume in one second ${ }^{[8]}$. Each of these are measured in liters and are reported as percent predicted for the patient ${ }^{[8]}$. The FVC is a measure of air the lungs can hold and is highly dependent on the amount of force used by the patient in early expiration. It is generally reduced in both obstructive and restrictive conditions. The FEV1 is a measure of how easily air flows through the lungs and reflects airflow in the larger airways ${ }^{[8,33]}$. COPD patients often have narrowing and inflammation of the airways and this hinders how fast the air can leave the lungs. This in turn leads to a fall in FEV1 ${ }^{[8]}$. If FEV1 is decreased disproportionately to the FVC a diagnosis of COPD is made ${ }^{[8]}$. To check if the decrease is disproportionate theFEV1/FVC ratio is calculated and a ratio of $<0.7$ after bronchodilator is considered a diagnosis of COPD ${ }^{[8]}$. Vesna Cukic found that in patients with COPD there is a decrease in FEV1 and FVC during the follow up period which indicates that airflow limitation is progressive but in patients taking regular therapy during exacerbations, both FVC and FEV1 are statistically significant more in comparison with patients not taking full therapy ${ }^{[32]}$.

Chunlal Chen et al. in 2016 demonstrated that early COPD patients with lung hyperinflation are associated with poorer lung functions but better bronchodilator responsiveness. ${ }^{[34]}$. They also found that patients without lung hyperinflation generally exhibited milder COPD and the change in FVC was the most significant factor associated with lung hyperinflation ${ }^{[34]}$. Recently it has been found that a specific subtype of COPD is classified by the forced vital capacity (FVC) ${ }^{\text {[9] }}$. Chunlan Chen et al. in 2018 conducted a study on 1329 patients with COPD and concluded that patients with COPD can be classified by a new category method according to before bronchodilator (BDD) and after bronchodilator (ABD) forced vital capacity. Patients with inconsistent FVC ie. I-FVC presented a higher residual volume and higher total lung capacity and had higher asthma and exacerbation frequencies [9]. I-FVC group demonstrated better responsiveness to bronchodilator evaluated both by FEV1 and FVC among groups ${ }^{[9]}$.

Airway mucus hypersecretion in COPD patients results in outcomes such as rapid decline in lung functions, poor quality of life and a higher rate of acute exacerbations, hospitalizations and mortality ${ }^{[2]}$. A 12 year follow up study of 1757 males and 1291 females revealed the relationship between chronic phlegm and FEV1 decline. In this study men and women show FEV1 decline of $4.5 \mathrm{ml} /$ year and $1.7 \mathrm{ml} /$ year respectively ${ }^{[2,6]}$.

A study by $G$ Donaldson has shown that patients with moderate to severe COPD who suffered frequent exacerbations experienced a significantly greater decline in FEV1 of 40ml/year and peak expiratory flow rate (PEFR) of $2.91 / \mathrm{min} /$ year than patients who had infrequent exacerbations ${ }^{[3]}$. PEFR is the maximum flow that occurs at any given point in time during the FVC. Normal peak flow averages to 9 to 10L/second. Decrease in PEFR reflect non specific mechanical problems ${ }^{[33]}$.

It is known that airway clearance techniques improve lung functions by enhancing mucus clearance. We have seen earlier that chronic cough and sputum production are common features of COPD and have a significant impact on quality of life ${ }^{[10]}$. Airway clearance techniques aid in secretion clearance but the use of ACT for COPD should take into consideration the presence of bronchiectasis, the amount of sputum produced, the degree of sputum produced, the degree of airflow obstruction and the presence of lung recoil ${ }^{[10]}$.

In COPD patients mucociliary transport is impaired and when this happens a secondary clearance mechanism known as two phase gas liquid flow plays an essential role in improving mucus ${ }^{[10]}$. Most of the airway clearance techniques make use of this mechanism to move the mucus towards the mouth ${ }^{[10]}$. As seen earlier when air flows over a thick mucus layer, a shear force is developed on the surface layer and when this shear force exceeds the surface tension in the mucus layer, the mucus begins to move in the direction of the airflow ${ }^{[10,16,29,17]}$. The effects of two phase gas liquid flow are enhanced when gravity is able to assist [10]. This principle is used in ELTGOL technique to aid in mucus clearance.

A study by Kylie Hill et al. done to check the effect of ACTs in patients experiencing an acute exacerbation of COPD concluded that ACTs did not improve resting lung functions but the application of 5minutes of continuous 
chest wall percussion reduced FEV1 by 7.3\%. [35] Mihalton et al. found that a device that spreads vibratory pneumatic signals in the bronchial tree during relaxed exhalation improves mucus clearance and thus improves FEV1, FEV1/FVC ratio and this device can be used in COPD patients with chronic bronchitis ${ }^{[14]}$.

As we can see that pulmonary functions are severely affected in COPD and airway clearance techniques can improve functions by aiding in mucus clearance. Hence ELTGOL, an airway clearance technique, is also useful in clearing mucus and improving pulmonary functions in patients with COPD.

\section{Conclusions}

The present study provides promising results in improving pulmonary function tests in patients with moderate to severe COPD.

Hence, we can accept our alternate hypothesis which stated that ELTGOL will be effective in improving pulmonary functions in patients with COPD after two weeks of intervention.

On the basis of the result we can conclude that ELTGOL is effective in improving pulmonary functions in patients with moderate to severe COPD.

\section{List of abbreviations}

GOLD - Global Initiative of Obstructive Lung Disease

COPD - Chronic Obstructive Lung Disease

ELTGOL - Slow Expiration with Glottis Open in Lateral

Decubitus position

WHO - World Health Organization

$\mathrm{NIH}$ - National Institute of Health

PFT - Pulmonary Function Test

FVC - Forced Vital Capacity

FEV1 - Forced Expiratory Volume in 1 Second

FEV1/FVC - Forced Expiratory Volume in Second/Forced Vital Capacity ratio

PEFR - Peak Expiratory Flow Rate

PIFR - Peak Inspiratory Flow Rate

ACT - Airway Clearance Technique

FRC - Functional Residual Capacity

RV - Residual Volume

\section{References}

1. Yash Pal Munjal. API Textbook of Medicine. Jaypee Brothers 9(2):1711

2. Pan-Wen Tian, Fu-Qiang. Clinical significance of airway mucus hypersecretion in Chronic Obstructive Pulmonary Disease. Journal of Translational International Medicine 2015;3(3):89-92

3. Donaldson GC, Seemungal TAR, Bhowmik A, Wedzicha JA. Relationship between exacerbation frequency and lung function decline in Chronic Obstructive Pulmonary Disease. Thorax 2002;57:847852

4. Nitin Y Bhatt, Karen L Wood. What defines abnormal lung function in older adults with Chronic Obstructive Disease. Drugs Ageing 2008;25(9):717-728

5. Sudeep Salvi, Anil Kumar $\mathrm{G}$ et al. The burden of chronic respiratory disease and their heterogenicity across the states of India: The Global Burden of disease study 1990-2016. Lancet Global Health 2018;6(8):1362-1364
6. Sherman CB, Xu X, Speizer PE, Ferris BG Jr, Weiss ST, Dockery DW. Longitudinal lung function decline in subjects with respiratory symptoms. Am Rev Respir Dis 1992;146:855-9

7. Vestbo J, Prescott E, Lange P. Association of chronic mucus hypersecretion with FEV1 decline and chronic obstructive pulmonary disease morbidity. Am J Respir Crit Care Med 1996;153:1530-1535

8. Kristina L Bailey. The importance of the assessment of pulmonary function in COPD. Med Clin North Am. 2012;96(4):745-752

9. Chunlal Chen, Ying He et al. A specific subtype of chronic obstructive pulmonary disease classified by Forced Vital Capacity. J Thoraci Dis 2018;10(12):65476565

10. Anne E Holland, Brenda M Button. Is there a role for airway clearance techniques in chronic obstructive pulmonary disease 2006;3:83-91

11. Franceso D’ Abrosca, Barbara Garabelli, Gloria Savio et al. Comparing airway clearance techniques in chronic obstructive pulmonary disease and bronchiectasis: positive expiratory pressure or temporary positive expiratory pressure? A retrospective study. Braz J Phys Ther 2017.

12. Elizabeth Westerdhal, Christian Osadnik. Airway clearance techniques for patients with acute exacerbations of chronic obstructive pulmonary disease, physical therapy practice in Sweden. Chronic Respiratory Disease 2019;16:1-8

13. Ada Gracie Gastadi. Flutter device review: Effects of secretions and pulmonary functions. J Nov Physiother. 2016;6(3):1-6

14. Mihaltan F, Morin L et al. Evaluation of an innovative airway clearance technology in comparison to manual chest physiotherapy in acute exacerbation of chronic obstructive pulmonary disease (COPD). Am J Respir Crit Care Med 2019, 199

15. Metka Kodric et al. The Effectiveness of Bronchial Drainage Technique (ELTGOL) in COPD exacerbations. Respirology 2009;14:424-428.

16. Conroy Wong, Cameroon Sullivan, Lata Jayram. ELTGOL airway clearance in bronchiectasis: laying the bricks of evidence. Eur Respir J 2018;51:1-3

17. Fernanda C Lanza et al. Expiratory reserve volume during slow expiration with glottis opened in infralateral decubitus position (ELTGOL) in chronic pulmonary disease: Technique Description and Reproducibility. Respiratory Care 2005;60(3):406-411.

18. Martins JA, Domela de Andrade et al. Effect of ELTGOL on mucus clearance in stable patients with chronic bronchitis. Respir Care 2012;57(3):420-426

19. Munzo G, de Gracia J, Buxo M et al. Long term effect of airway clearance in bronchiectasis: a randomised placebo-controlled trial. Eur Respir J 2018;51:1701926

20. Herrero-Cortina B et al. Short term effects of three slow expiratory airway clearance techniques in patients with bronchiectasis: a randomised crossover trail. Physiotherapy 2015;07:005: 1-7

21. Richa Rajeev Agarwal, Md. Abu Shaphe, Chacko George, Anurag Vats. A comparison of Flutter device and active cycle of breathing technique in acute exacerbation of chronic obstructive pulmonary disease patients. Indian Journal of Physiotherapy and Occupational Therapy 2010;4(3):60-64 
22. Bellone A, Lascioli R, Raschi S, Guzzi L, Adone R. Chest physical therapy in patients with acute exacerbations of chronic bronchitis; effectiveness of three methods. Arch Phys Med Rehabil 2000;81(5):558-560

23. Schoni MH. Autogenic drainage: a modern approach to physiotherapy in cystic fibrosis. J R Soc Med 1989;82(16):32-37

24. Pryor JA. Physiotherapy for airway clearance in adults. Eur Respir J 1999;14(6):1418-1424.

25. Agnew JE, Bateman JR, Pavia D, Clarke SW. A model for assessing bronchial mucus transport. J Nucl Med 1984;25(2):170-176.

26. Guimaraes FS, Molo VJ, Menezes SL et al. Effect of ELTGOL and Flutter VRPI on dynamic and static pulmonary volumes and on the secretion clearance of patients with bronchiectasis. Rev Bras Fisioter 2012;16:108-113

27. Guimaraes FS, Lopes AJ et al. ELTGOL acutely improves airway clearance and reduces static pulmonary volumes in adult cystic fibrosis patients. J Phys Ther Sci 2014;28:813-816

28. Postiaux G, Lens E et al. Efficacite de l'expiration lente totalr glotte ouverete en decubitus infralateral (ELTGOL): sur la toiletle en peripherie de l'arbre tracheobronchique. Ann Kinesther 1990;17(3):87-99

29. Kim CS, Iglesias AJ, Sackner MA. Mucus clearance by two phase gas liquid flow mechanism asymmetric periodic flow model. J Appl Physiol 1987;61:959-971.

30. Van der Schans CP. Bronchial mucus transport. Respir Care 2007;52:1150-1156

31. Voets PJ, Van Helvroot HA. The role of equal pressure points in understanding pulmonary disease. Adv Physical Educ 2013;37:266-267

32. Rodney G Benjamin, Gillette A Chapman, Chong S Kim, Marvin A Sackner. Removal of bronchial secretions by two-phase gas liquid transport. Chest 1989;95:658-664

33. Mcllwaine $\mathrm{M}$, Bradley $\mathrm{J}$ et al. Personalising airway clearance in chronic lung disease. Eur Respir Rev 2017;26:160086

34. Vesna Cukic, Valdimir Lovre, Adia Ustamujic. The changes of pulmonary function in COPD during fouryear period. Mat Soc Med 2013;25(2):88-92

35. Ellen Hillegass. Essentials of cardiopulmonary physical therapy. Elsevier 3(10):377

36. Chunlal Chen, Wanhua Jian, Yi Gao et al. Early COPD patients with lung hyperinflation associated with poorer lung function but better bronchodilator responsiveness. International Journal of COPD 2016;11:2519-2526

37. Kylie Hill, Shane Patman, Dina Brooks. Effects of airway clearance techniques in patients experiencing an acute exacerbation of chronic obstructive pulmonary disease. A systemic review. Chronic Respiratory Disease 2010;7(1):9-17

38. Ada Clarice Gastadi. Flutter Device Review: Effects on secretion and pulmonary function. J Nov Physiother 2016;6:3

39. Saowanee W, Suwanee J et al. Efficacy of pursed lip breathing with forced expiration and active cycle of breathing technique on pulmonary mucus clearance in healthy subjects. J Phys. Ther. Sci 2010;22:247-254
40. Binu Babu, Jincy Ealias, Abhilash V. pursed lip breathing exercise - A self management approach towards shortness of breath 2016;1(1):41-45

41. Üzmezoğlu B, Altıay G, Özdemir L et al. The Efficacy of Flutter ${ }^{\circledR}$ and Active Cycle of Breathing Techniques in Patients with Bronchiectasis: A Prospective, Randomized, Comparative Study. Turk Thorac J. 2018;19(3):103-109

42. Wada et al. Effects of aerobic training combined with respiratory muscle stretching on the functional exercise capacity and thoracoabdominal kinematics in patients with COPD: A randomized controlled trial. International Journal of COPD 2016;11:2691-2700.

43. Kerti et al. The relationship between exercise capacity and different functional markers in pulmonary rehabilitation for COPD. International Journal of COPD. 2018;13:717-724

44. Coen AC, Leo MA, Richard RN. Diaphragm adaptations in patients with COPD. Respiratory research 2018, 9-12

45. Cano Porras D et al. Comparison between the phase angle and phase shift parameters to assess thoracoabdominal asynchrony in COPD patients. J. Appl Physiol 2017;122:1106-1113.

46. Alves GS. et al. Breathing pattern and thoracoabdominal motion during exercise in chronic obstructive pulmonary disease. Braz J. Med Biol. Res. 2008;4(11):945-950

47. Kaminsky et al. Effect of yoga breathing (pranayama) on exercise tolerance in patients with chronic obstructive pulmonary disease: A randomised controlled trial. The Journal of alternative and complimentary medicine 2017;23:696-704

48. Daitx RB. et al. Limited utility of Kinesio taping in the physiotherapy treatment for patients with chronic obstructive pulmonary disease exacerbation. Physiotherapy Theory and Practice 2018, 1423658

49. Leonardo F, Manoela H, Rodrigo DM et al. Expiratory and Inspiratory muscle strength in subjects with COPD: Systemic review. Respiratory Care. 2014;59(9):13811388

50. Rocha et al. The manual diaphragmatic release technique improves diaphragmatic mobility, inspiratory capacity and exercise capacity in people with chronic obstructive pulmonary disease: a randomised trial. 2015;61:182-189. 